Provided for non-commercial research and education use. Not for reproduction, distribution or commercial use.

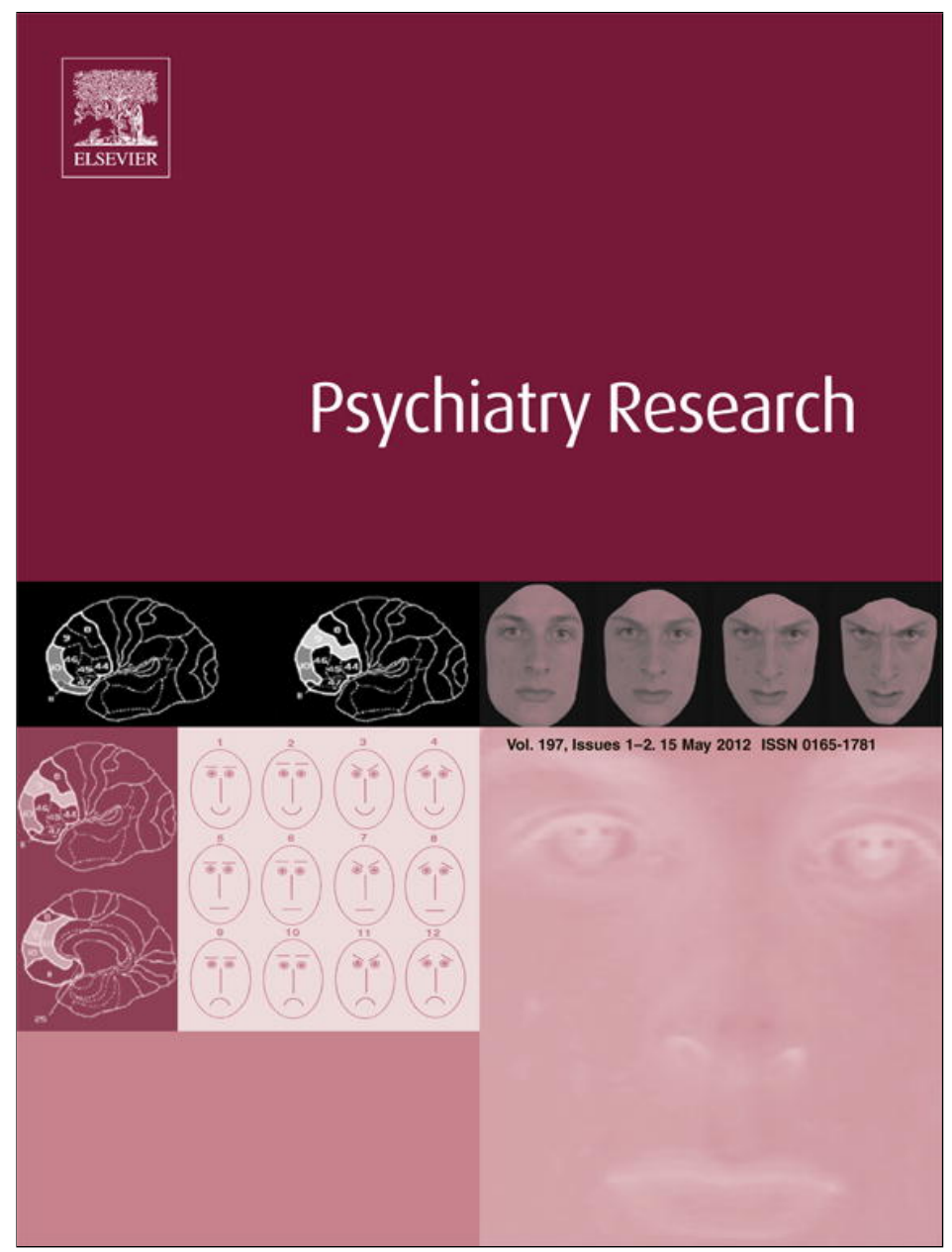

This article appeared in a journal published by Elsevier. The attached copy is furnished to the author for internal non-commercial research and education use, including for instruction at the authors institution and sharing with colleagues.

Other uses, including reproduction and distribution, or selling or licensing copies, or posting to personal, institutional or third party websites are prohibited.

In most cases authors are permitted to post their version of the article (e.g. in Word or Tex form) to their personal website or institutional repository. Authors requiring further information regarding Elsevier's archiving and manuscript policies are encouraged to visit:

http://www.elsevier.com/copyright 


\title{
Differentiating PTSD symptomatology with the MMPI-2-RF (Restructured Form) in a forensic disability sample
}

\author{
Martin Sellbom ${ }^{\text {a,* }}$, Tayla T.C. Lee ${ }^{\mathrm{b}}$, Yossef S. Ben-Porath ${ }^{\mathrm{b}}$, Paul A. Arbisi ${ }^{\mathrm{c}}$, Roger O. Gervais ${ }^{\mathrm{d}}$ \\ a Department of Psychology, The University of Alabama, Tuscaloosa, AL 35487-0348, USA \\ b Department of Psychology, Kent State University, Kent, OH 44242-0001, USA \\ c Minneapolis VA Medical Center, One Veterans Drive, Minneapolis, MN 55417, USA \\ ${ }^{\mathrm{d}}$ Neurobehavioural Associates, 210, 17010-103 Avenue, Edmonton, AB, Canada T5S 1K7
}

\section{A R T I C L E I N F O}

\section{Article history:}

Received 22 March 2011

Received in revised form 29 January 2012

Accepted 6 February 2012

\section{Keywords:}

PTSD

MMPI-2-RF

Personality

Temperament

Assessment

\begin{abstract}
A B S T R A C T
The current study was designed to explore models of assessing various forms of Post-Traumatic Stress Disorder (PTSD) symptomatology that incorporate both broad and more narrowly focused affective markers. We used broader markers of demoralization, negative activation, positive activation, and aberrant experiences to predict global PTSD scores, whereas more narrowly focused markers of positive and negative affect were used to differentiate between PTSD symptom clusters. A disability sample consisting of 347 individuals undergoing medico-legal psychological evaluations was used for this study. All participants completed symptom measures of PTSD and the Minnesota Multiphasic Personality Inventory-2 (MMPI-2) (from which MMPI-2-RF scores were derived). The results indicated that demoralization was the best individual predictor of PTSD globally, and that more narrowly focused MMPI-2-RF Specific Problems scales provided a differential prediction of PTSD symptom clusters. Theoretical and practical implications of these findings are discussed within contemporary frameworks of internalizing personality and psychopathology.
\end{abstract}

(C) 2012 Elsevier Ireland Ltd. All rights reserved.

\section{Introduction}

Post-Traumatic Stress Disorder (PTSD) is a mental disorder resulting from exposure to an emotionally traumatic event perceived by the individual as potentially causing serious physical harm or death (American Psychiatric Association, 2000). In the current edition of the Diagnostic and Statistical Manual of Mental Disorders (DSM-IVTR; APA, 2000), symptoms of PTSD are rationally organized into three broad categories. The first cluster of re-experiencing symptoms involves emotional, cognitive, behavioral, or physical reactions to internal or external cues attributed to exposure to the traumatic stressor. The second cluster of PTSD symptoms involves both avoidance of stimuli associated with the trauma and generalized numbing of affective responsivity. The third cluster, hyperarousal, entails symptoms related to increased arousal beginning after the traumatic event.

PTSD has high rates of comorbidity with other mental disorders, particularly with mood, other anxiety, and substance-related disorders.

* Corresponding author at: Department of Psychology, 348 Gordon Palmer Hall, The University of Alabama, Tuscaloosa, AL 35487-0348, USA. Tel.: +1 205348 9936; fax: +1 2053488648

E-mail addresses: msellbom@ua.edu (M. Sellbom), taylatclee@gmail.com (T.T.C. Lee), ybenpora@kent.edu (Y.S. Ben-Porath), Paul.Arbisi@va.gov (P.A. Arbisi), rgervais@shaw.ca (R.O. Gervais).
For example, in a large community sample, Kessler et al. (1995) estimated the prevalence of lifetime PTSD was $7.8 \%$ and indicated, of those individuals with a lifetime history of PTSD, $88.3 \%$ of men and $79 \%$ of women had a lifetime history of at least one other Axis I disorder. Further, previous research has indicated the rates of comorbidity are more extreme in individuals currently receiving treatment for PTSD, with a demonstrated rate of current PTSD being comorbid with another Axis I disorder of $92 \%$ in one large community anxiety disorders clinic (Brown et al., 2001).

One method of accounting for this extraordinary rate of comorbidity has been to explore alternative conceptualizations of PTSD and other disorders using results from factor analytic studies in an attempt to delineate the structure of common mental disorders. Such analyses have yielded a broad internalizing psychopathology dimension, consisting of two discrete sub-factors labeled "anxious-misery" and "fear," which have been supported both via genotypic vulnerability (e.g., Kendler et al., 2003) and phenotypic covariance models (e.g., Krueger and Markon, 2006). Watson (2005) has alternatively labeled the anxious-misery sub-dimension the "distress" disorders, in order to emphasize the pervasive subjective distress characteristic of the disorders linked to the anxious-misery sub-dimension. Major Depression, Dysthymic Disorder, and Generalized Anxiety Disorder have been linked to the anxious-misery sub-dimension, whereas Social Phobia, Specific Phobia, Agoraphobia, and Panic Disorder have been linked to the fear sub-dimension (Watson, 2005). 
PTSD was not included in the original studies examining the structure of common mental disorders (e.g., Krueger, 1999). However, Watson (2005) argued that PTSD should be conceptualized as a distress disorder, as indicated by results of exploratory factor analyses using large representative samples from the United States (Cox et al., 2002) and Australia (Slade and Watson, 2006). In both studies, PTSD symptoms loaded more highly on the anxious-misery sub-dimension, although in both samples factor loadings for PTSD were lower than those of other disorders. As these results suggest that PTSD symptoms are heterogeneous, Watson (2005) left open the possibility that certain PTSD symptoms might load on the fear dimension, rather than distress dimension. Subsequently, a confirmatory factor analytic study by Forbes et al. (2010) provided empirical support for subsets of PTSD symptoms having differential loadings on the anxious-misery and fear dimensions. Symptoms related to re-experiencing, active avoidance, and exaggerated fear responses loaded strongly onto the fear dimension, whereas symptoms related to numbing, irritability, and sleep and concentration difficulties loaded onto the anxious-misery dimension in a large sample of injury survivors 3-, 12-, and 24-months after admission to specialized trauma services.

Another method of accounting for comorbidity between disorders that has been pursued in previous research is to relate them to underlying shared temperament factors (Clark, 2005). Temperament markers are hypothesized to represent potential genetic diatheses for a range of adult personality characteristics, as well as psychopathological conditions that develop in response to sufficient environmental stressors, providing a framework of associations between temperament, personality, and psychopathology. As such, this approach seeks to account for comorbidity between disorders by relating them to underlying shared temperament factors, while allowing for differentiation through the identification of unique associations for different disorders with lower level temperament sub-facets (e.g., Krueger et al., 1996; Clark, 2005).

For internalizing disorders, this line of research on temperament markers has focused largely on aspects of the Two-Factor model of affect involving positive activation and negative activation (Watson and Tellegen, 1985; Tellegen et al., 1999). Characterized by negative affective arousal (e.g., "afraid" and "angry"), negative activation has been demonstrated to be a marker of non-specific distress common to both anxiety and mood disorders (Watson et al., 1988, 2005, 2006). Alternatively, positive activation, characterized by positive affective arousal (e.g., "excited" and "delighted"), appears to be a marker of depressive disorders and social phobia.

Sellbom et al. (2008a) proposed and showed empirical support for an elaborated hierarchical structure designed to provide more specific and differentiated affective personality markers of internalizing disorders. Specifically, the authors proposed to separate a generalized distress component from the Positive and Negative Activation dimensions based on Tellegen and colleagues (Tellegen, 1985; Tellegen et al., 1999; Watson et al., 1999) conceptual framework for affect, in an effort to increase the specificity of measurement for negative affect other than general maladjustment. Demoralization, representing general maladjustment and dysphoric mood, was hypothesized to be reflected in Tellegen's (1985) happiness-unhappiness dimension. This dimension of affect describes a higher-order, bipolar structure of the shared and inversely related hedonic features of negative and positive activation (Watson and Tellegen, 1985; Tellegen et al., 1999; Watson et al., 1999), reflecting variations in hedonic valence (e.g., happy and sad), rather than the distinctive variations in arousal that are captured by negative and positive activation. Working from this model and using a series of confirmatory factor analyses, Sellbom et al. (2008a) demonstrated the elaborated temperament model containing negative activation, positive activation, and demoralization provided a better fit to the data than the alternative model containing only negative and positive activation. Additionally, results indicated demoralization was the primary marker of distress disorders, negative activation was the primary marker of fear disorders, and positive activation was a specific marker of depression and social phobia.

Relatively few studies of the relations between psychopathology and temperament have focused on or included PTSD. Watson et al. (2005) found that measures of negative activation were associated with the endorsement of traumatic memories in a sample of college students. These authors also demonstrated that dysphoric PTSD symptoms were related to negative activation in a sample of gulf war veterans, although the relative pattern of relations suggested that negative activation was more strongly associated with symptoms of depression and generalized anxiety than with PTSD symptoms. These results were supported by Gamez et al. (2007) who demonstrated that current best estimate diagnoses of PTSD were related to markers of negative activation in a large sample of Gulf War veterans. Additionally, the overall pattern of correlations suggested that PTSD symptoms were more similar in underlying personality predictors to distress disorders when compared to fear disorders, with the exception of a unique association between PTSD symptoms and a marker of eccentric perceptions.

The PTSD literature just reviewed links the disorder with the broadband internalizing dimension of psychopathology, in addition to temperament domains of demoralization and negative activation. However, Clark (2005) suggested that examination of facets of the basic temperament dimensions could facilitate identification of symptom subsets related to various disorders. Such research would require the use of measures that allow for a multi-level (broad- and narrowband) assessment of psychopathology.

The Minnesota Multiphasic Personality Inventory-2-Restructured Form (MMPI-2-RF; Ben-Porath and Tellegen, 2008; Tellegen and Ben-Porath, 2008) could provide the broad and narrow-band measurement needed to assist in differentiating PTSD from other mental disorders. Specifically, the MMPI-2-RF contains the Restructured Clinical scales (Tellegen et al., 2003) and the more narrowly focused Specific Problems scales (Ben-Porath and Tellegen, 2008), both of which are conceptually relevant to the PTSD literature and needed levels of measurement just discussed. The RC scales assess mid-level traits, including demoralization, negative activation, and positive activation (Tellegen et al., 2003). Further, previous research with the RC scales has demonstrated they map well onto normal personality/ temperament models (Sellbom and Ben-Porath, 2005; Sellbom et al., 2008b) and are congruent with current conceptualizations of mood and anxiety disorders (Sellbom et al., 2008a), including PTSD (Wolf et al., 2008). The Specific Problems scales assess facets of the broader personality domains represented by the Restructured Clinical scales (Ben-Porath and Tellegen, 2008), making them ideal candidates for facilitating identification of specific subsets of symptom for various disorders (as suggested by Clark, 2005). A list and brief description of the 13 MMPI-2-RF scales included in this study is provided in Table 1.

The current study sought to examine the ability of both broad and narrow-band personality markers, like those represented in the MMPI-2-RF scales just described, to predict and differentiate between subsets of PTSD symptoms. Our first goal was to examine broadband markers of PTSD using MMPI-2-RF scales indexing demoralization (RCd), positive activation (Low Positive Emotions [RC2]), negative activation (Dysfunctional Negative Emotions [RC7]), and aberrant experiences (RC8). We hypothesized that RCd, reflecting demoralization, rather than RC7, a marker of negative activation, would be the primary marker underlying global PTSD symptomatology. Such a finding would replicate the results of Sellbom et al. (2008a), and support that PTSD is best conceptualized as a distress disorder (e.g., Watson, 2005). Further, previous research has suggested PTSD is uniquely associated with eccentric perceptions when compared to other internalizing disorders (Gamez et al., 2007). We therefore hypothesized that RC8 - a measure of aberrant experiences (which includes dissociative experiences relevant to PTSD; Tellegen and Ben-Porath, 2008) - would be a 
Table 1

Labels, abbreviations, and brief description of MMPI-2-RF Scales used in the current investigation.

\begin{tabular}{|c|c|c|}
\hline Scale & Abbreviation & Brief description \\
\hline Demoralization & $\mathrm{RCd}$ & $\begin{array}{l}\text { Emotional distress, unhappiness, and } \\
\text { dissatisfaction }\end{array}$ \\
\hline Low Positive Emotions & $\mathrm{RC} 2$ & Lack of positive emotional responsiveness \\
\hline $\begin{array}{l}\text { Dysfunctional Negative } \\
\text { Emotions }\end{array}$ & RC7 & $\begin{array}{l}\text { Maladaptive anxiety, fear, anger/ } \\
\text { irritability, guilt }\end{array}$ \\
\hline Aberrant Experiences & RC8 & Unusual/bizarre perceptions or thoughts \\
\hline $\begin{array}{l}\text { Helplessness/ } \\
\text { Hopelessness }\end{array}$ & HLP & $\begin{array}{l}\text { Belief that goals cannot be reached or } \\
\text { problems solved }\end{array}$ \\
\hline Self-Doubt & SFD & Lack of confidence, feelings of uselessness \\
\hline Inefficacy & NFC & Indecisiveness, belief that one is inefficacious \\
\hline Stress/Worry & STW & $\begin{array}{l}\text { Stress reactivity, obsessive rumination, } \\
\text { difficulty with time pressure }\end{array}$ \\
\hline Anxiety & AXY & $\begin{array}{l}\text { Pervasive anxiety, frights, frequent } \\
\text { nightmares, intrusive ideation }\end{array}$ \\
\hline Anger Proneness & ANP & $\begin{array}{l}\text { Becoming easily angered, impatient with } \\
\text { others, low frustration tolerance }\end{array}$ \\
\hline $\begin{array}{l}\text { Behavior-Restricting } \\
\text { Fears }\end{array}$ & BRF & $\begin{array}{l}\text { Fears that significantly inhibit normal } \\
\text { activities }\end{array}$ \\
\hline Multiple Specific Fears & MSF & Fears of blood, fire, thunder, etc. \\
\hline Social Avoidance & SAV & Avoiding and/or not enjoying social events \\
\hline
\end{tabular}

secondary marker of the disorder. Indeed, Wolf et al. (2008) found that RC8 significantly differentiated combat veterans with PTSD from those who did not meet criteria for this disorder.

Our second goal was to examine associations between lowerorder symptom clusters of PTSD as defined by the DSM-IV-TR (APA, 2000) and narrowband MMPI-2-RF Specific Problems scales assessing facets of demoralization and negative activation/emotionality. We expected that facets of demoralization (Helplessness/Hopelessness [HLP], Self-Doubt [SFD], and/or Inefficacy [NFC]) would be particularly useful in identifying PTSD symptoms of a dysphoric nature (contained within the Avoidance and Hypersousal clusters, cf. Simms et al., 2002). Additionally, we hypothesized that three MMPI-2-RF scales assessing facets of negative activation or emotionality (i.e., Stress/Worry [STW], Anxiety [AXY], Anger Proneness [ANP]) would be associated with Re-experiencing, Avoidance, and Hyperarousal symptom clusters. Specifically, STW and AXY, measures related to stress reactivity and worry (Tellegen and Ben-Porath, 2008), were expected to be associated with all three DSM symptom clusters. Anger Proneness, assessing the anger component of negative activation, was expected to be associated only with Hyperarousal symptoms. Furthermore, partially based on Forbes et al. (2010), we hypothesized that the two fear scales (Behavior-Restricting Fears [BRF] and Multiple Specific Fears [MSF]) would only be associated with Hyperarousal symptoms. Finally, as lack of social support has been demonstrated to be one of the strongest predictors of development of PTSD symptoms after traumatic exposures (Brewin et al., 2000), we hypothesized that scores on the MMPI-2-RF Social Avoidance (SAV) scale would contribute to the prediction of avoidance-related PTSD symptoms.

\section{Method}

\subsection{Participants and procedures}

Our sample was extracted from a large archival database of disability claimants referred for medico-legal psychological evaluations at an independent practice in Edmonton, Alberta, Canada. A total of 395 individuals who had been administered the MMPI-2 and the three PTSD measures used in this study were selected from this large database. These individuals had been referred for assessment of traumatic stress or other psychological difficulties due to trauma following workplace injuries, motor vehicle collisions, or other traumatic exposures.

Because over-reporting of psychological problems is commonplace in these settings, and because we wanted a sample with genuine psychological problems, we excluded those who engaged in inconsistent responding or symptom over-reporting. More specifically, we first consulted three symptom validity tests (Word Memory Test [Green, 2003], Test of Memory Malingering [Tombaugh, 1996], and Computerized Assessment of Response Bias [Allen et al., 1997]), with failure on these SVTs being determined according to cut offs recommended in their respective manuals. ${ }^{1}$ Specific cut-off scores or other failure criteria are not presented here in the interests of maintaining test security and to deter coaching (Youngjohn, 1995). Participants who failed any of these tests were excluded, which totaled $48(12 \%)$ of participants. Among these 48 individuals, $66.1 \%$ failed one test, $25.0 \%$ failed two tests, and $8.9 \%$ failed three tests. Moreover, in accordance with MMPI-2-RF interpretative guidelines (Ben-Porath and Tellegen, 2008), participants were also excluded based on MMPI-2-RF Validity scales. These exclusionary criteria included for scores on Cannot Say $\geq 18$, Variable Response Inconsistency or True Response Inconsistency $\geq 80 \mathrm{~T}$, Frequency $=120 \mathrm{~T}$, and FrequencyPsychopathology $\geq 100 \mathrm{~T}$ (Ben-Porath and Tellegen, 2008). This procedure excluded an additional $52(13 \%)$ participants. ${ }^{2,3}$

The final sample consisted of 159 men and 136 women, ages 19 to 65 years $(M=40.29$, S.D. $=10.86)$, who had an average of 12.01 (S.D. $=2.48)$ years of education. Although ethnicity and race were not formally recorded, the sample was representative of the population demographics in the province of Alberta, as described by Gervais et al. (2011). Worker's Compensation Board (WCB) (65\%) and other medicolegal $(28.5 \%)$ referrals constituted the majority of the referral sources. Primary psychiatric diagnoses were PTSD (54.8\%), depressive disorder (24.7\%), and chronic pain (18.0\%), with orthopedic injuries or other conditions constituting the remaining sample. Diagnoses were based on DSM-IV (APA, 1994) or DSM-IV-TR (APA, 2000) criteria, following an extensive clinical interview, psychological test data (which included the MMPI-2, but not the MMPI-2-RF scales used in this study), and accompanying medical or other third party documentation. In terms of PTSD Criterion A level traumatic event, $66 \%$ of the sample reported work-related events, whereas $30 \%$ had been involved in a motor vehicle accident during which significant fear or helplessness was experienced.

\subsection{Measures}

\subsubsection{MMPI-2-RF}

All participants were administered the MMPI-2 from which MMPI-2-RF (BenPorath and Tellegen, 2008) scores can be derived, as the entire 338 items scores on the MMPI-2-RF are contained in the MMPI-2. Internal consistencies (Cronbach's alpha) of MMPI-2-RF scale scores in the current study were 0.90 (RCd), 0.78 (RC2), 0.88 (RC7), 0.74 (RC8), 0.63 (HLP), 0.73 (SFD), 0.75 (NFC), 0.67 (STW), 0.69 (AXY), 0.77 (ANP), 0.60 (BRF), 0.68 (MSF), and 0.83 (SAV). Extensive evidence supporting validity for scores on these scales is provided in the MMPI-2-RF Technical Manual (Tellegen and Ben-Porath, 2008).

\subsubsection{DAPS}

The Detailed Assessment of Posttraumatic Stress (DAPS; Briere, 2001) is a 104item standardized instrument containing three specific scales that assess reexperiencing, avoidance, and hyperarousal symptoms of PTSD, as well as other scales reflecting features or sequela of the disorder. All claimants endorsed at least one of the 13 types of trauma covered by the DAPS or specified another trauma event that may not have been represented under the 13 potential events exposure. Item leve data for the scales were not available to calculate internal consistency reliability for DAPS scale scores in the current study due to the nature of the archival database. The DAPS Technical Manual (Briere, 2001) provides substantial support for the reliability (mean $\alpha=0.83$ ) and validity of the instrument's scale scores in trauma-exposed adults in clinical, community, and university samples.

\subsubsection{DTS}

The 17-item Davidson Trauma Scale (DTS; Davidson et al., 1997) was developed and validated utilizing several different samples of trauma-exposed individuals and provides a total score and three subscales scores assessing intrusion, avoidance/numbing, and hyperarousal symptoms. Due to the archival nature of this database, item level data were not available in the current study to calculate internal consistency reliability. Davidson et al (1997) reported in the development sample the DTS demonstrated good test-retest reliability (mean $r=0.86$ ) and internal consistency (mean $\alpha=0.99$ ) for its total and facet scale scores. Moreover, the measure demonstrated concurrent validity as individuals with a PTSD diagnosis (per structured interview) had substantially higher scores than those without a PTSD diagnosis (Davidson et al., 1997).

2.2.4. IES

The 15-item Impact of Events Scale (IES; Horowitz et al., 1979) contains a total symptom score, as well as two subscales assessing intrusion and avoidance. Due to the archival nature of this database, item level data were not available in the current study to calculate internal consistency reliability for this measure. However, as

${ }^{1}$ Almost $86 \%$ of participants had scores on all three symptom validity tests, and all participants had scores on at least two.

${ }^{2}$ We recognize that $25 \%$ is a very high exclusionary rate, but these rates are consistent with malingering rates in similar settings (e.g., Mittenberg et al., 2002). Because this study focused on genuine psychological problems, we took a conservative approach.

${ }^{3}$ The MMPI-2-RF FBS-r and RBS scales have been found sensitive to over-reporting in this type of setting, and using these scales would have resulted in an additional 14 participants being excluded. We elected not to use these criteria, however, because they are not recommended to be used to invalidate the entire MMPI-2-RF profile. Moreover, the pattern of results was identical with these exclusions. 
reviewed by Joseph (2000), numerous studies in varying samples with differing traumatic exposures have supported acceptable levels of both internal consistency and test-retest reliability, as well as the validity of IES scale scores.

\subsection{Data analyses}

We conducted structural equation modeling (SEM) that focused on examining MMPI-2-RF scales in predicting latent variables representing PTSD symptoms globally, as well as individual PTSD symptom clusters (i.e., re-experiencing, avoidance, and hyperarousal). This approach confers a number of advantages, including (a) that the latent factor variance will reflect what these indicators have in common, which means that the latent factor will predict a large proportion of variance in each indicator, (b) capitalizing on multiple indicators for the same constructs to increase confidence in construct measurement from different perspectives, and (c) the ability to separately model random measurement error in addition to indicator-specific variances, which yields less biased parameters in the structural model, and ones that are more representative of the population. For PTSD symptomatology, we specified a model in which latent representations of the three individual PTSD symptom clusters served as indicators for a second-order global PTSD factor. The various cluster subscales from IES, DTS, and DAPS served as indicators for their respective PTSD (i.e., reexperiencing, avoidance, and hyperarousal) latent variable. ${ }^{4}$

The first SEM model concerned the prediction of global PTSD symptomatology. Here, RCd, RC2, RC7, and RC8 served as predictors. We specified a latent regression model in which the second-order PTSD latent variable was regressed onto the Restructured Clinical scale predictors. The second SEM analysis was focused on the prediction of the various PTSD symptom clusters for which we used the correlated three-factor model. We used the hypothesized Internalizing Specific Problems scales as predictors, with two additions: RC8 was also included to predict Re-Experiencing and the SAV Specific Problems scale was included to predict Avoidance. See Fig. 2 for the model that includes all hypothesized paths. These latent variables were simultaneously regressed onto the hypothesized MMPI-2-RF predictors just mentioned.

The models were evaluated using Mplus 5.21 (Muthén and Muthén, 2007). The model parameters were estimated using maximum likelihood with robust scaling to account for potential violation of multivariate normality. Model fit was evaluated using the Satorra-Bentler $\chi^{2}$ statistic, Comparative Fit Index (CFI), Tucker-Lewis Index (TLI), Root Mean Square Error of Approximation (RMSEA) with 90\% confidence intervals, and Standardized Root Mean Square Residual (SRMR). Chi square statistics are heavily influenced by sample size (Kline, 2011), and therefore, other indices are better estimates of fit in a large sample (Bentler, 2007). CFI and TLI values greater than .90 are indicative of acceptable fit (Hu and Bentler, 1999). The RMSEA and SRMR values up to 0.05 indicate good fit, between 0.06 and 0.08 indicate adequate fit, and RMSEA $\geq 0.10$ indicate poor fit (Hu and Bentler, 1999; Kline, 2011). Missing data, which was minimal, was handled via the full information maximum likelihood procedure. We evaluated individual standardized parameter estimates (i.e., $\beta$ s) based on statistical significance and, more importantly, effect size estimates $(0.10=$ small, $0.30=$ medium, and $0.50=$ large effect size; see Cohen, 1988).

\section{Results}

Means and standard deviations, as well as estimates of skewness and kurtosis, for all these measures based on the current sample are displayed in Table 2. Distributions for all variables used in the current study approximated normal (i.e., skew $<2$ and kurtosis $<7$ ). After the exclusionary criteria mentioned earlier were applied, complete data were available for all scales in both samples.

We first tested a model that focused on predicting the global second-order latent PTSD variable on which the DAPS, DTS, and IES total scores loaded. We regressed this variable onto RCd, RC2, RC7, and RC8. The model had acceptable fit, SB- $\chi^{2}=132.19$, d.f. $=43$, $p<0.001, \mathrm{CFI}=0.95, \mathrm{TLI}=0.92, \mathrm{RMSEA}=0.078$ (90\% CI 0.062, 0.094), $\mathrm{SRMR}=0.048$. The multiple $R^{2}$ in predicting global PTSD was 0.46 $(p<0.001)$. Fig. 1 provides a graphical presentation of this model and Table 3 lists the standardized weights for each of the Restructured Clinical scales in predicting PTSD. As evident from this table, RCd was

\footnotetext{
${ }^{4}$ For the Hyperarousal PTSD latent variable, there were only two manifest markers, DTS Hyperarousal and DAPS Hyperarousal, as the IES does not have a hyperarousal subscale.

${ }^{5}$ A thoughtful reviewer questioned whether the PTSD measures were too similar to be multiple indicators of the same construct. We do not believe that these measures are isomorphic for the following reasons: The DTS has 17 items, the DAPS has 104 items, and the IES has 15 items (for two subscales only). The items covering similar areas are not worded in an identical fashion. Moreover, the observed correlations (i.e., $r s=0.69$ to 0.79 for Intrusion subscales; $0.55-0.63$ for Avoidance subscales; and 0.59 for two Hyperarousal subscales) are substantially lower than what would be expected if these tests measured identical constructs.
}

Table 2

Means, standard deviations, skewness, and kurtosis for MMPI-2-RF and PTSD Scales.

\begin{tabular}{|c|c|c|c|c|}
\hline Scale & $M$ & S.D. & Skewness & Kurtosis \\
\hline 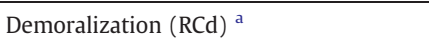 & 64.49 & 11.74 & -0.264 & -0.669 \\
\hline Low Positive Emotions (RC2) ${ }^{a}$ & 63.69 & 13.53 & 0.376 & -0.467 \\
\hline Dysfunctional Negative Emotions (RC7) a & 59.08 & 13.30 & 0.071 & -0.676 \\
\hline Aberrant Experiences (RC8) a & 57.32 & 11.63 & 0.528 & 0.137 \\
\hline Helplessness/Hopelessness (HLP) a & 59.69 & 14.38 & 0.348 & -0.801 \\
\hline Self-Doubt (SFD) a & 60.17 & 11.77 & 0.050 & -1.199 \\
\hline Inefficacy (NFC) ${ }^{a}$ & 56.65 & 11.68 & 0.204 & -0.822 \\
\hline Stress/Worry (STW) ${ }^{a}$ & 58.37 & 12.13 & 0.179 & -0.866 \\
\hline Anxiety $(\mathrm{AXY})^{\mathrm{a}}$ & 69.44 & 17.95 & 0.036 & -1.101 \\
\hline Anger Proneness (ANP) ${ }^{a}$ & 57.19 & 12.36 & 0.320 & -0.875 \\
\hline Behavior-Restricting Fears (BRF) ${ }^{a}$ & 55.10 & 12.99 & 0.879 & 0.230 \\
\hline Multiple Specific Fears (MSF) ${ }^{a}$ & 49.11 & 8.66 & 1.016 & 1.750 \\
\hline Social Avoidance (SAV) a & 54.85 & 12.20 & 0.547 & -0.629 \\
\hline DAPS Post-Traumatic Stress Total a & 85.28 & 17.43 & -0.951 & -0.431 \\
\hline DAPS Re-Experiencing ${ }^{\text {a }}$ & 86.42 & 23.41 & -0.185 & -0.995 \\
\hline DAPS Avoidance ${ }^{a}$ & 82.68 & 20.98 & -0.171 & -0.922 \\
\hline DAPS Hyperarousal a & 88.49 & 19.51 & -0.689 & -0.456 \\
\hline DTS total score & 78.68 & 28.80 & -0.468 & -0.451 \\
\hline DTS intrusion & 22.15 & 10.50 & -0.353 & -0.742 \\
\hline DTS Avoidance/Numbing & 30.11 & 13.16 & -0.374 & -0.584 \\
\hline DTS Hyperarousal & 26.45 & 9.40 & -0.630 & -0.416 \\
\hline IES total PTSD score & 43.08 & 17.48 & -0.743 & 0.104 \\
\hline IES Intrusion & 21.98 & 9.82 & -0.598 & -0.471 \\
\hline IES Avoidance & 21.13 & 9.39 & -0.528 & -0.313 \\
\hline
\end{tabular}

Note. DAPS = Detailed Assessment of Post-Traumatic Stress, DTS = Davidson Trauma Scale, IES = Impact of Events Scale.

a Expressed in T-scores.

the only scale that contributed significantly to this prediction, with a moderate effect size. ${ }^{6}$

The next model included the prediction of three latent variables representing the individuals PTSD symptom clusters - Re-experiencing, Avoidance, and Hyperarousal. These latent variables were simultaneously regressed onto the hypothesized Specific Problems scales. Fig. 2 provides a graphical representation of the overall model, which had acceptable fit, SB- $\chi^{2}=218.37$, d.f. $=79, p<0.001, C F I=0.93$, $\mathrm{TLI}=0.90, \quad \mathrm{RMSEA}=0.077 \quad(90 \%$ CI $0.065,0.090), \quad \mathrm{SRMR}=0.038$ Table 3 shows the standardized weights for each of the MMPI-2-RF scales predicting the three latent PTSD symptom clusters. In terms of predicting the Re-experiencing cluster, the multiple $R^{2}$ was .38 $(p<0.001)$. An examination of the standardized weights indicated that only AXY contributed significantly to this prediction and was associated with a large effect size. Unexpectedly, RC8, which was included in the study specifically for the purpose of predicting these types of PTSD symptoms, did not contribute significantly. For the prediction of the latent variable representing PTSD Avoidance symptoms, the multiple $R^{2}$ was $0.42(p<0.001)$. AXY contributed most substantially to this prediction with a moderate effect. Also, as expected, SFD and SAV added significant unique predictions to this model, albeit with small effect sizes. For predicting the PTSD Hyperarousal latent variable, the multiple $R^{2}$ was $0.28(p<0.001)$. AXY and ANP contributed significantly to this prediction, all with small effect sizes.

Finally, we estimated a pruned SEM model in which we only retained the significant structural parameters from the model just described. All other structural parameters were fixed to zero. This model, which had acceptable fit, SB- $\chi^{2}=258.65$, d.f. $=90, p<0.001, \mathrm{CFI}=0.94$, $\mathrm{TLI}=0.90, \mathrm{RMSEA}=0.080(90 \% \mathrm{CI} 0.069,0.091), \mathrm{SRMR}=0.046$, is depicted in Fig. 3. AXY $(\beta=0.62, p<0.001)$ continued to be a significant predictor of Re-Experiencing; SFD $(\beta=0.19, p<0.001)$, AXY $(\beta=0.47$, $p<0.001)$, and $\operatorname{SAV}(\beta=0.11, p<0.005)$ remained significant predictors of Avoidance; and AXY $(\beta=0.42, p<0.001)$ and ANP $(\beta=0.28$, $p<0.001$ ) predicted Hyperarousal symptoms.

${ }^{6}$ Unstandardized regression weights with associated standard errors and $p$-values for all scales in all models are available upon request from the first author. 


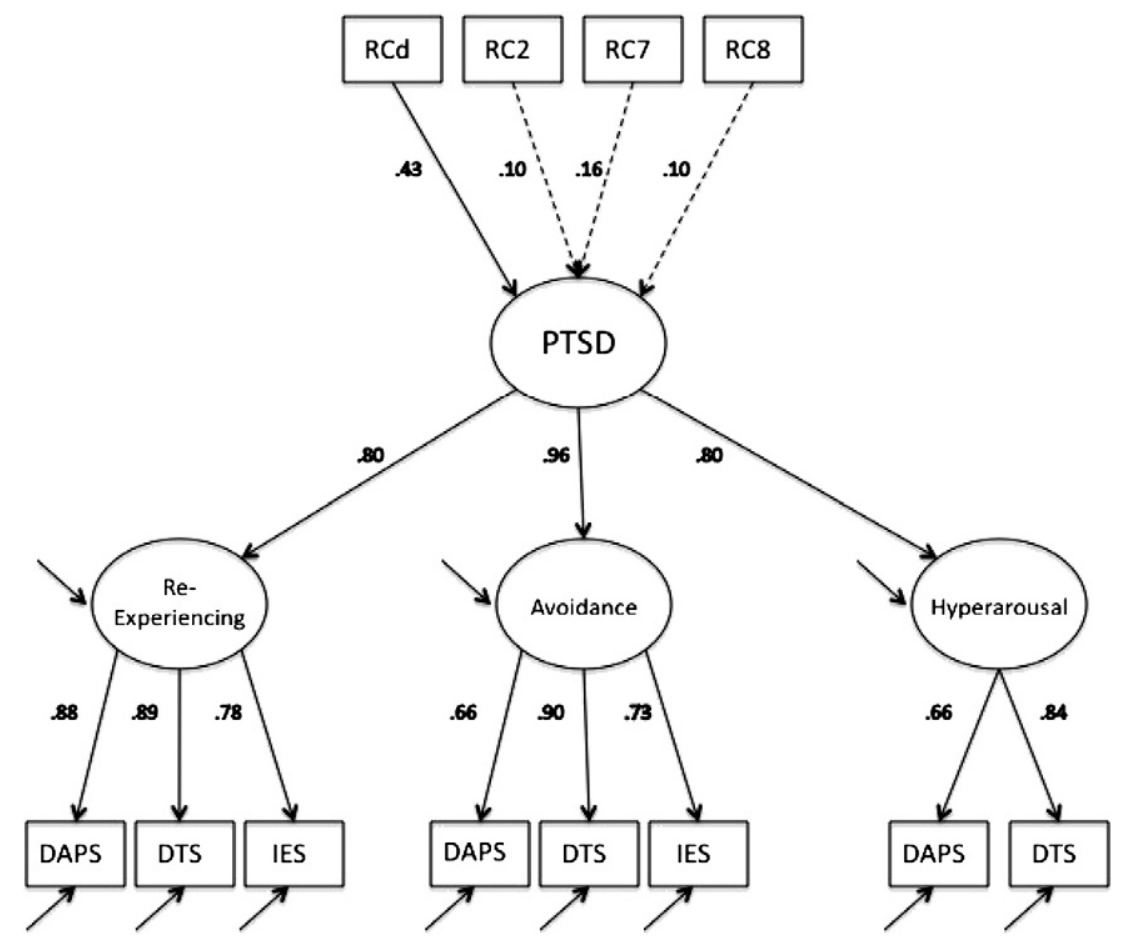

Fig. 1. Structural regression model for the prediction of global PTSD latent $(N=295)$. Intercorrelations between predictor variables are not illustrated due to space and readability, but are available in Appendix A. All factor loadings are statistically significant $(p<0.001)$, and parameters are standardized based on both predictor and criterion variables. Broken parameters are not statistically significant. RCd $=$ Demoralization, RC2 $=$ Low Positive Emotions, RC7 $=$ Dysfunctional Negative Emotions, RC8 $=$ Aberrant Experiences, DAPS $=$ Detailed Assessment of Post-Traumatic Stress, DTS = Davidson Trauma Scale, IES = Impact of Events Scale.

\section{Discussion}

The goal of the current study was to explore the utility of the MMPI-2-RF in differentiating symptoms of PTSD with scales indexing broad- and narrow-band markers of affectivity. Overall, the findings generally conformed to our hypotheses; the MMPI-2-RF Restructured Clinical scales accounted for a substantial amount of variance in global PTSD symptomatology, whereas internalizing Specific Problems scales predicted variance in the specific clusters of PTSD symptoms.

Table 3

Standardized parameter estimates for MMPI-2-RF predictions in PTSD global and cluster models $(N=295)$.

\begin{tabular}{|c|c|c|c|c|c|c|c|c|}
\hline & \multirow{2}{*}{\multicolumn{2}{|c|}{$\begin{array}{l}\text { PTSD Total } \\
R^{2}=0.46\end{array}$}} & \multirow{2}{*}{\multicolumn{2}{|c|}{$\begin{array}{l}\text { Re-experiencing } \\
R^{2}=0.38\end{array}$}} & \multirow{2}{*}{\multicolumn{2}{|c|}{$\frac{\text { Avoidance }}{R^{2}=0.42}$}} & \multirow{2}{*}{\multicolumn{2}{|c|}{$\begin{array}{l}\text { Hyperarousal } \\
R^{2}=0.28\end{array}$}} \\
\hline & & & & & & & & \\
\hline & $\beta$ & $P$ & $B$ & $p$ & $\beta$ & $p$ & $B$ & $p$ \\
\hline $\mathrm{RCd}$ & 0.43 & $<0.001$ & & & & & & \\
\hline $\mathrm{RC} 2$ & 0.10 & 0.096 & & & & & & \\
\hline RC7 & 0.16 & 0.066 & & & & & & \\
\hline RC8 & 0.10 & 0.070 & 0.05 & 0.280 & & & & \\
\hline HLP & & & & & 0.09 & 0.071 & 0.03 & 0.562 \\
\hline SFD & & & & & 0.16 & 0.003 & 0.13 & 0.111 \\
\hline NFC & & & & & 0.06 & 0.290 & -0.02 & 0.671 \\
\hline STW & & & -0.06 & 0.360 & 0.03 & 0.673 & 0.10 & 0.165 \\
\hline AXY & & & 0.62 & $<0.001$ & 0.42 & $<0.001$ & 0.25 & 0.005 \\
\hline ANP & & & & & & & 0.17 & 0.048 \\
\hline BRF & & & & & & & 0.07 & 0.139 \\
\hline MSF & & & & & & & -0.07 & 0.138 \\
\hline SAV & & & & & 0.10 & 0.035 & & \\
\hline
\end{tabular}

Note. RCd = Demoralization, RC2 = Low Positive Emotions, RC7 = Dysfunctiona Negative Emotions, RC8 = Aberrant Experiences, HLP $=$ Helplessness/Hopelessness, SFD $=$ Self-Doubt, NFC = Inefficacy, STW $=$ Stress/Worry, AXY $=$ Anxiety, ANP = Anger Proneness, BRF $=$ Behavior-Restricting Fears, MSF $=$ Multiple Specific Fears and SAV $=$ Social Avoidance.
Consistent with our hypotheses, the MMPI-2-RF measure of demoralization (i.e., RCd) was most strongly associated with global PTSD symptomatology. When RCd, RC2, RC7, and RC8 were considered in a model, RCd provided the largest unique contribution in the prediction of global PTSD symptoms. Overall, these results lend further support to the conceptualization of PTSD as a distress disorder from the temperament perspective, consistent with the extant literature on PTSD, and not surprising given the large dysphoria component associated with the disorder (Simms et al., 2002). Specifically, this result is congruent with previous research indicating PTSD is primarily a distress disorder (cf. Watson, 2005; and see Cox et al., 2002; Slade and Watson, 2006; but see Forbes et al., 2010 for a different perspective) and that demoralization is the major shared temperament dimension among distress disorders (Sellbom et al., 2008a). Further, other studies (e.g., Watson et al., 2005; Gamez et al., 2007) have also indicated that nonspecific distress represents a broad personality marker for PTSD symptoms when assessed globally, in addition to when the prediction of specific symptoms is examined (Marshall et al., 2010).

However, as indicated earlier, Watson (2005) suggested certain PTSD symptom clusters could be differentially associated with distress and fear dimensions, an idea which has been supported by the results of at least one confirmatory factor analytic study in injury patients (Forbes et al., 2010). Our results also provide partial support for this hypothesis, and although global PTSD symptoms were best predicted by demoralization, some specific PTSD symptoms in the current study were more strongly associated with negative activation markers. Indeed, the best marker of all three clusters of PTSD symptoms in the current study was the AXY scale - a measure of intrusive ideation (including nightmares) and hypervigilance (Tellegen and Ben-Porath, 2008). Anger Proneness, another negative activation marker, was also a significant contributor to the prediction of Hyperarousal, albeit to a smaller degree. Furthermore, the association between SAV and Avoidance symptoms demonstrated in the current study is consistent with findings relating PTSD and social detachment (Gamez 


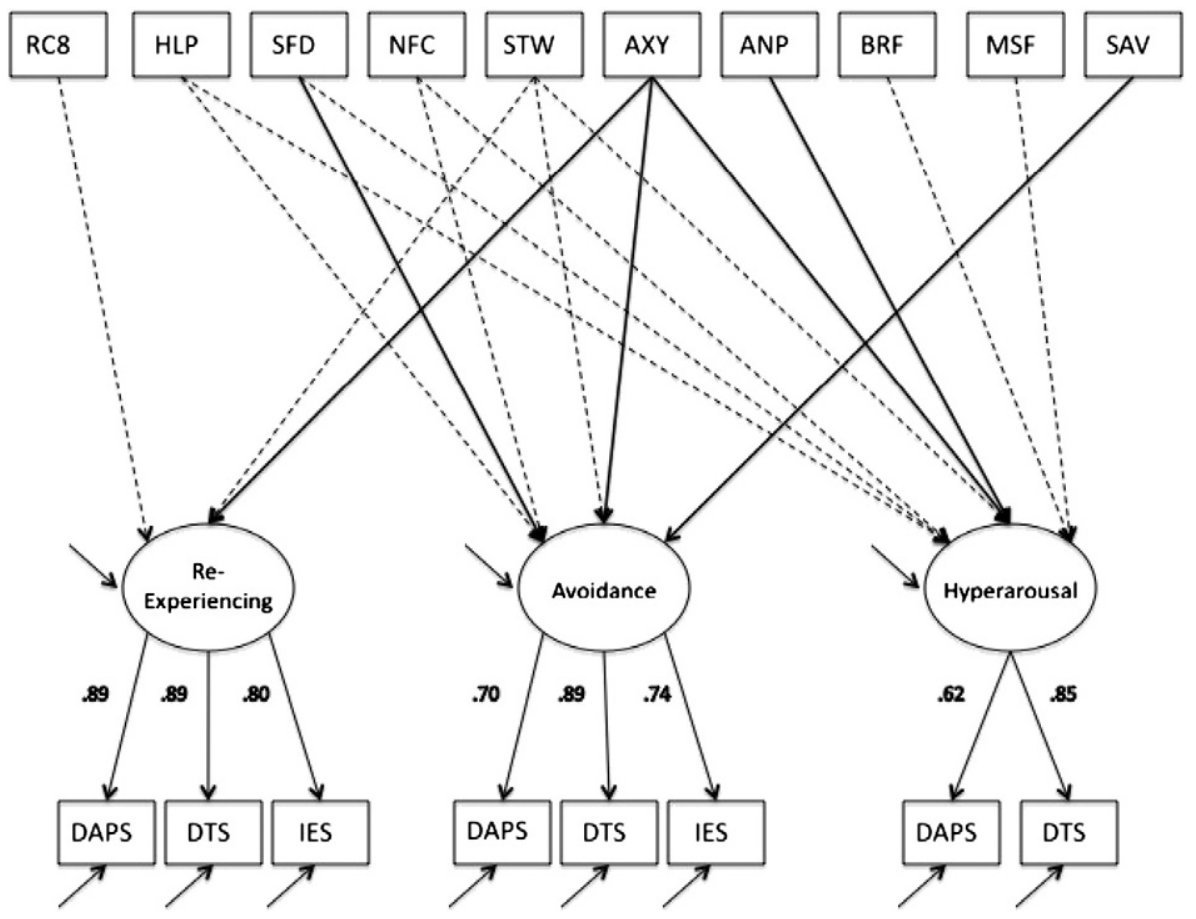

Fig. 2. Hypothesized structural regression model for the prediction of PTSD latent symptom $(N=295)$. Intercorrelations between predictor variables are not illustrated due to space and readability, but are available in Appendix A. All factor loadings are statistically significant $(p<0.001)$, and parameters are standardized based on both predictor and criterion variables. Broken lines represent parameters that are not statistically significant. Table 3 presents information about predictor beta weights. RC8 $=$ Aberrant Experiences, HLP $=$ Helplessness/Hopelessness, SFD = Self-Doubt, NFC = Inefficacy, STW = Stress/Worry, AXY = Anxiety, ANP = Anger Proneness, BRF $=$ Behavior-Restricting Fears, MSF $=$ Multiple Specific Fears, and SAV = Social Avoidance, DAPS = Detailed Assessment of Post-Traumatic Stress, DTS = Davidson Trauma Scale, IES = Impact of Events Scale.

et al., 2007), as well as meta-analytic results suggesting decreased social support is substantially associated with the development of PTSD (Brewin et al., 2000).
Some findings in the current study did not conform to a priori hypotheses. The expectation that RC8, included as a measure of aberrant experiences, would add to the prediction of PTSD symptoms

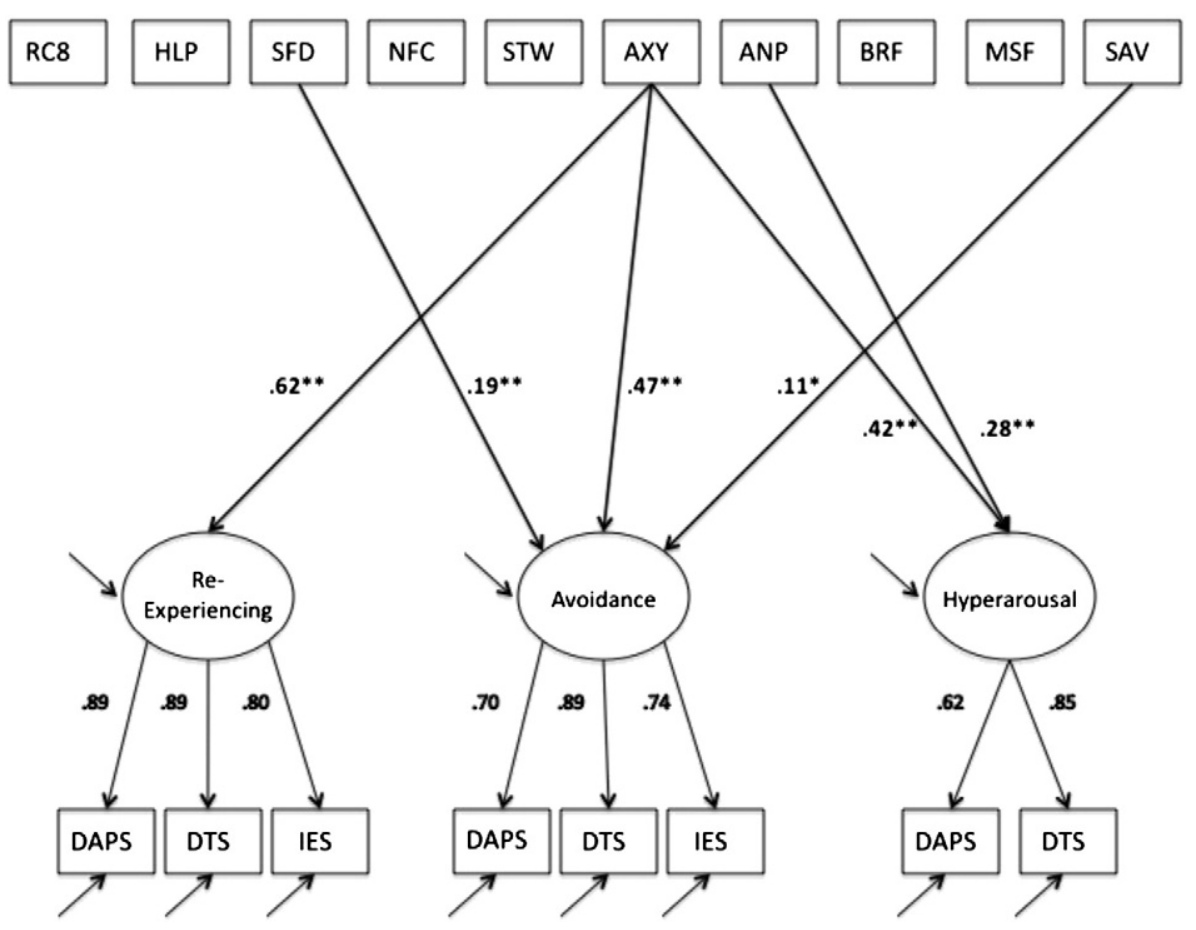

Fig. 3. Final pruned structural regression model for the prediction of PTSD latent symptom $(N=295)$. Intercorrelations between predictor variables are not illustrated due to space and readability, but are available in Appendix A. All factor loadings are statistically significant $(p<0.001)$, and parameters are standardized based on both predictor and criterion variables. Parameters not shown in this model were fixed to zero. RC8 = Aberrant Experiences, HLP $=$ Helplessness $/$ Hopelessness, SFD $=$ Self-Doubt, NFC $=$ Inefficacy, STW = Stress/Worry, AXY = Anxiety, ANP = Anger Proneness, BRF = Behavior-Restricting Fears, MSF = Multiple Specific Fears, and SAV $=$ Social Avoidance, DAPS $=$ Detailed Assessment of Post-Traumatic Stress, DTS = Davidson Trauma Scale, IES = Impact of Events Scale. ${ }^{*} p<0.01,{ }^{* *} p<0.001$. 
(particularly re-experiencing symptoms) was not supported. There are two possible reasons for this outcome. First, although previous studies have found that RC8 differentiates between male combat veterans with and without PTSD (Wolf et al., 2008), these researchers did not consider RC8 in a model that simultaneously included RCd, $\mathrm{RC} 2$, and RC7. Second, RC8 may not be a good measure of intrusive symptoms of PTSD as it is contains a large number of overt and extreme psychotic symptoms. As such, a less extreme measure of eccentric perceptions, like that demonstrated to be a significant predictor of PTSD symptoms by Gamez et al. (2007), may have been more appropriate to assess these symptoms. Thus, we suggest that future studies examine these hypotheses, using alternative markers of re-experiencing symptoms in a model that also includes measures of negative activation, positive activation, and demoralization. Lastly, in the current study some internalizing Specific Problems scales, such as Inefficacy - a demoralization facet, and Stress/Worry - a negative activation facet - did not add uniquely to the prediction of any PTSD symptoms. This outcome suggests these scales may better differentiate other distress disorders, such as Generalized Anxiety Disorder (cf. Sellbom and Gervais, 2010), though a direct comparison will need to be conducted empirically in future research.

The current findings also have implications for applied assessment of PTSD symptoms with the MMPI-2-RF. In individuals with significant trauma histories, elevated scores on RCd could indicate possible PTSD, in addition to numerous other distress disorders (e.g., Major Depressive Disorder and Generalized Anxiety Disorder). Past and current results suggest that other Restructured Clinical scales can assist in further differentiation. RC2 is uniquely tied to depressive symptomatology (e.g., Tellegen et al., 2006; Sellbom et al., 2008a), and not PTSD, as evident in the current study. Thus, the absence of RC2 elevation when RCd is elevated would further increase the possibility of PTSD symptoms; however, the presence of an RC2 elevation would not rule it out given potential comorbidity with depression. In terms of the Specific Problems scales, the AXY scale is strongly and uniquely associated with numerous PTSD symptoms, especially those that are distinct from other distress disorders (e.g., Re-experiencing, Hyperarousal). The Specific Problems scales SFD and SAV could be considered for measuring Avoidance symptoms, whereas ANP could be used for assessing Hyperarousal symptoms, with the caution that this measure was associated with a small to moderate effect size (see Fig. 3) in the regression models.

There are limitations to this study that warrant discussion. First only self-report measures were used to assess PTSD symptoms and we were therefore limited to examining dimensional symptom domains rather than specific diagnostic criteria. In addition, shared method variance may have artificially inflated the associations between PTSD symptoms and MMPI-2-RF scales. However, because we were more interested in patterns of associations than absolute correlational magnitudes, this limitation might not have substantial impact in the current investigation. Furthermore, this mono-methodology also limits the potential for understanding basic psychopathology processes, as both trait and PTSD symptoms are assessed the same way. Thus, future studies need replicate these findings using other measurement modalities.

Second, we were unable to examine alternative models of PTSD symptomatology and it is possible in the current study the utility of the MMPI-2-RF scales in predicting different types of PTSD symptomatology was clouded by the use of the rationally derived DSM-IV-TR three-cluster model of symptoms. This is a particular limitation given previous research suggesting strong support for a four-factor model of symptoms (e.g., Simms et al., 2002). Unfortunately, we only had access to scale data for this sample because of the archival nature of the database, and there is no established method of measuring any of the four-factor PTSD symptom structures with the DAPS, DTS, and IES. We, nonetheless, believe that our current approach is worthwhile. The three factor model as indicated by the IES, DTS, and DAPS subscales actually demonstrated adequate model fit in the current sample when a measurement model was specified prior to including the structural elements $\left(\chi^{2}=70.00\right.$, d.f. $=17, p<0.001$, $\mathrm{CFI}=0.96$, RMSEA $=0.081$ ). The three-factor model is still the one used to cluster symptoms in the DSM-IV-TR. Future studies should attempt to replicate these findings with other measures from which four-factor structures can be derived.

Finally, our sample was comprised primarily of disability claimants seen for psychological assessment following work-related injuries or trauma. Although PTSD claims are common in these settings and our sample is thus representative of a highly relevant population in which to examine the current research questions, the findings of the current study may not generalize to samples in which PTSD has resulted from other forms of trauma. Furthermore, despite using careful methods to screen out potential malingering, it is possible that some malingerers remained in the sample and it is impossible to determine the degree to which such response styles may have influenced the results. In light of these limitations, future research needs to replicate these findings in clinical samples of non-compensation seeking nature, and where other types of traumatic experiences (e.g., sexual assault) are more prevalent.

\section{Appendix A. Supplementary data}

Supplementary data to this article can be found online at doi:10. 1016/j.psychres.2012.02.003.

\section{References}

Allen III, L.M., Conder, R.L., Green, P., Cox, D.R., 1997. CARB '97: Manual for the Computerized Assessment of Response Bias. CogniSyst, Durham, NC.

American Psychiatric Association, 1994. Diagnostic and Statistical Manual of Mental Disorders, 4th ed. American Psychiatric Association, Washington, DC

American Psychiatric Association, 2000. Diagnostic and Statistical Manual of Mental Disorders, 4th ed., text revision. American Psychiatric Association, Washington, DC

Ben-Porath, Y.S., Tellegen, A., 2008. MMPI-2-RF (Minnesota Multiphasic Personality Inventory-2-Restructured Form): Manual for Administration and Scoring. University of Minnesota Press, Minneapolis, MN

Bentler, P.M., 2007. On tests and indices for evaluating structural models. Personality and Individual Differences 42, 825-829.

Brewin, C.R., Andrews, B.A., Valentine, J.D., 2000. Meta-analysis of risk factors for posttraumatic stress disorder in trauma-exposed adults. Journal of Consulting and Clinical Psychology 68, 748-766.

Briere, J., 2001. DAPS: Detailed Assessment of Posttraumatic Stress. Psychological Assessment Resources, Inc., Odessa, Florida.

Brown, T.A. Campbell, LA., Lehman, C.L, Grisham, J.R., Mancill, R.B., 2001. Current and lifetime comorbidity of the DSM-IV anxiety and mood disorders in a large clinical sample. Journal of Abnormal Psychology 110, 585-599.

Clark, L.A., 2005. Temperament as a unifying basis for personality and psychopathology Journal of Abnormal Psychology 114, 505-521.

Cohen, J., 1988. Statistical Power for the Behavioral Sciences. Lawrence Erlbaum Associates, Hillsdale, NJ.

Cox, B.J., Clara, I.P., Enns, M.W., 2002. Posttraumatic stress disorder and the structure of common mental disorders. Depression and Anxiety 15, 168-171.

Davidson, J.R.T., Book, S.W., Colket, J.T., Tupler, L.A., Roth, S., David, D., Hertzberg, M. Mellman, T., Beckham, J.C., Smith, R.D., Davison, R.M., Katz, R., Feldman, M.E., 1997. Assessment of a new self-rating scale for posttraumatic stress disorder: THE Davidson Trauma Scale. Psychological Medicine 27, 153-160.

Forbes, D., Parslow, R., Creamer, M., O'Donnell, M., Bryant, R., McFarlane, A., Silove, D. Shaley, A., 2010. A longitudinal analysis of posttraumatic stress disorder symptoms and their relationship with fear and anxious-misery disorders: implications for DSM-V. Journal of Affective Disorders 127, 147-152.

Gamez, W., Watson, D., Doebbeling, B.N., 2007. Abnormal personality and the mood and anxiety disorders: implications for structural models of anxiety and depression. Journal of Anxiety Disorders 21, 526-539.

Gervais, R.O., Wygant, D.B., Sellbom, M., Ben-Porath, Y.S., 2011. Associations between symptom validity test failure and scores on the MMPI-2-RF Validity and Substantive Scales. Journal of Personality Assessment 93, 508-517. doi:10.1080/00223891. 2011.594132.

Green, P., 2003. Green's Word Memory Test for Windows: User's manual. Green's Publishing, Edmonton.

Horowitz, M., Wilner, N., Alvarez, W., 1979. Impact of event scale: a measure of subjective stress. Psychosomatic Medicine 41, 209-218.

Hu, L., Bentler, M., 1999. Cutoff criteria for fit indexes in covariance structure analysis: conventional criteria versus new alternatives. Structural Equation Modeling $6,1-55$.

Joseph, S., 2000. Psychometric evaluation of Horowitz's Impact of Event Scale: a review. Journal of Traumatic Stress 13, 101-113. 
Kendler, K.S., Prescott, C.A., Myers, J., Neale, M.C., 2003. The structure of genetic and environmental risk factors for common psychiatric and substance use disorders in men and women. Archives of General Psychiatry 60, 929-937.

Kessler, R.C., Sonnega, A., Bromet, E., Hughes, M., Nelson, C.B., 1995. Posttraumatic stress disorder in the National Comorbidity Survey. Archives of General Psychiatry 52, $1048-1060$.

Kline, R.B., 2011. Principles and Practice Of Structural Equation Modeling, 3rd Ed. Guilford Press, New York.

Krueger, R.F., 1999. The structure of common mental disorders. Archives of General Psychiatry 56, 921-926.

Krueger, R.F., Markon, K., 2006. Reinterpreting comorbidity: a model-based approach to understanding and classifying psychopathology. Annual Review of Clinical Psychology 2, 111-133.

Krueger, R.F., Capsi, A., Moffit, T.E., Silva, P.A., McGee, R., 1996. Personality traits are differentially linked to mental disorders: a multitrait-multidiagnosis study of an adolescent birth cohort. Journal of Abnormal Psychology 105, 299-312.

Marshall, G.N., Schell, T.L., Miles, J.N.V., 2010. All PTSD symptoms are highly associated with general distress: ramifications for the dysphoria symptom cluster. Journal of Abnormal Psychology 119, 126-135.

Mittenberg, W., Patton, C., Canyock, E.M., Conditt, D.C., 2002. Base rates of malingering and symptom exaggeration. Journal of Clinical and Experimental Neuropsychology 24, 270-279.

Muthén, L.K., Muthén, B.O., 2007. Mplus User’s Guide, Fifth Edition. Muthén \& Muthén, Los Angeles, CA.

Sellbom, M., Ben-Porath, Y.S., 2005. Mapping the MMPI-2 Restructured Clinical Scales onto normal personality traits: evidence of construct validity. Journal of Personality Assessment 85, 179-187.

Sellbom, M., Gervais, R.O., 2010, August. Differentiating Distress Disorder Symptomatology with the MMPI-2-RF Internalizing Scales, in: Ben-Porath, Y.S. (Chair), Reinforcing the link between practice and research with the MMPI-2-RF. Symposium presented at the 115th Annual Convention of the American Psychological Association, San Diego, CA.

Sellbom, M., Ben-Porath, Y.S., Bagby, R.M., 2008a. On the hierarchical structure of mood and anxiety disorders: confirmatory evidence and elaboration of a model of temperament markers. Journal of Abnormal Psychology 117, 576-590.

Sellbom, M., Ben-Porath, Y.S., Bagby, R.M., 2008b. Personality and psychopathology: mapping the MMPI-2 Restructured Clinical (RC) Scales onto the five factor model of personality. Journal of Personality Disorders 22, 291-312.

Simms, L.J., Watson, D., Doebbeling, B.N., 2002. Confirmatory factor analyses of posttraumatic stress symptoms in deployed and nondeployed veterans of the Gulf War. Journal of Abnormal Psychology 111, 637-647.
Slade, T., Watson, D. 2006. The structure of common DSM-IV and ICD-10 mental disorders in the Australian general population. Psychological Medicine 36, 1593-1600.

Tellegen, A., 1985. Structures of mood and personality and their relevance to assessing anxiety, with an emphasis on self-report. In: Tuma, A.H., Maser, J.D. (Eds.), Anxiety and the Anxiety Disorders. Lawrence Erlbaum Associates, Hillsdale, NJ, pp. 681-706.

Tellegen, A., Ben-Porath, Y.S., 2008. MMPI-2-RF (Minnesota Multiphasic Personality Inventory-2-Restructured Form): Technical Manual. University of Minnesota Press, Minneapolis, MN.

Tellegen, A., Watson, D., Clark, L.A., 1999. On the dimensional and hierarchical structure of affect. Psychological Science 10, 297-303.

Tellegen, A., Ben-Porath, Y.S., McNulty, J.L., Arbisi, P.A., Graham, J.R., Kaemmer, B., 2003. MMPI-2 Restructured Clinical (RC) Scales: Development, Validation, and Interpretation. University of Minnesota Press, Minneapolis, MN.

Tellegen, A., Ben-Porath, Y.S., Sellbom, M., Arbisi, P.A., McNulty, J.L., Graham, J.R., 2006. Further evidence on the validity of the MMPI-2 Restructured Clinical (RC) Scales: addressing questions raised by Rogers et al. and Nichols. Journal of Personality Assessment 87, 148-171.

Tombaugh, T.N., 1996. Test of Memory Malingering. Multi-Health Systems, Toronto.

Watson, D., 2005. Rethinking the mood and anxiety disorders: a quantitative hierarchical model for DSM-V. Journal of Abnormal Psychology 114, 522-536.

Watson, D., Tellegen, A., 1985. Toward a consensual structure of mood. Psychological Bulletin 98, 219-235.

Watson, D., Clark, L.A., Carey, G., 1988. Positive and negative affectivity and their relation to anxiety and depressive disorders. Journal of Abnormal Psychology 97, 346-353.

Watson, D., Gamez, W., Simms, L.J., 2005. Basic dimensions of temperament and thei relation to anxiety and depression: a symptom based perspective. Journal of Research in Personality 39, 46-66.

Watson, D., Wiese, D., Vaidya, J., Tellegen, A., 1999. The two general activation systems of affect: structural findings, evolutionary considerations, and psychobiological evidence. Journal of Personality and Social Psychology 76, 820-838.

Watson, D., Kotov, R., Gamez, W., 2006. Basic dimensions of temperament in relation to personality and psychopathology. In: Krueger, R.F., Tackett, J.L. (Eds.), Personality and Psychopathology. Guilford Press, New York, pp. 7-38.

Wolf, EJ Miller, M.W. Orazem, RJ. Weierich, M.R Castillo, D.T., Milford, J., Kaloupek, D.G., Keane, T.M., 2008. The MMPI-2 Restructured Clinical Scales in the assessment of posttraumatic stress disorder and comorbid disorders. Psychological Assessment $20,327-340$.

Youngjohn, J.R., 1995. Confirmed attorney coaching prior to neuropsychological evaluation. Assessment 2, 279-283. 